\title{
Synthetic Tumor-Associated Peptide Vaccine Therapy
}

National Cancer Institute

\section{Source}

National Cancer Institute. Synthetic Tumor-Associated Peptide Vaccine Therapy. NCI

Thesaurus. Code C116544.

A type of immunotherapy that uses a synthetic peptide to deliver tumor-specific antigens into the body in an effort to induce a targeted immune system response directed at the tumor. 